\title{
Enhanced dispersion by elastic turbulence in porous media
}

\author{
Christian Scholz ${ }^{1}$, Frank Wirner ${ }^{1}$, Juan Ruben Gomez-Solano ${ }^{1,2}$ and Clemens Bechinger ${ }^{1,2}$ \\ 1 2. Physikalisches Institut, Universität Stuttgart-Pfaffenwaldring 57, 70569 Stuttgart, Germany \\ 2 Max-Planck-Institut für Intelligente Systeme - Heisenbergstraße 3, 70569 Stuttgart, Germany
}

PACS 47.56. $+r-$ Flows through porous media

PACS 47.50.-d - Non-Newtonian fluid flows

PACS 47.50.Gj-Instabilities

\begin{abstract}
We experimentally investigate hydrodynamic dispersion in elastic turbulent flows of a semi-dilute aqueous polymer solution within a periodic porous structure at ultra-low Reynolds numbers $<10^{-3}$ by particle tracking velocimetry. Our results indicate that elastic turbulence can be characterized by an effective dispersion coefficient which exceeds that of Newtonian liquids by several orders of magnitude and grows non-linearly with the Weissenberg number Wi. Contrary to laminar flow conditions, the velocity field, and thus the shear rate, is not proportional to the flow rate and becomes asymmetric at high Wi.
\end{abstract}

Introduction. - Turbulent flow is characterized by unsteady velocity fields which suddenly vary in space and time. For Newtonian liquids, this regime is reached for high Reynolds numbers Re, where inertial effects dominate over viscous forces. In contrast, for viscoelastic fluids, turbulent flow at arbitrarily small Re numbers, usually referred to as elastic turbulence [1], can be observed. Such fluids are characterized by an elastic response, e.g. due to the entanglement and the dynamics of polymer chains [2]. The degree of elastic effects can be described by the dimensionless Weissenberg number $\mathrm{Wi}=\lambda \cdot \dot{\gamma}$, which quantifies the anisotropic polymer alignment in the presence of shear [3]. Here, $\lambda$ is the equilibrium polymer relaxation time and $\dot{\gamma}$ the shear rate of the flow. Accordingly, for $\mathrm{Wi} \rightarrow 0$ the response of the fluid to a sudden stress is purely viscous while for $\mathrm{Wi}>0$ an elastic short-time response is obtained. Since the polymer alignment not only depends on the instantaneous but also on the previous shear, this explains why a highly non-linear flow dependence on the shear strength is observed [2]. It has been experimentally demonstrated that above a critical Weissenberg number $\mathrm{Wi}_{c} \gtrsim 1$, elastic turbulence occurs [4]. Similar to inertial turbulence, this regime is characterized by an enhanced flow resistance and a power-law decay of the spectral power density $[1,5,6]$. This effect can be exploited to dramatically increase the mixing efficiency at small length scales and small Reynolds numbers $\left(\operatorname{Re}<10^{-4}\right)$ in microfluidic devices $[7,8]$.
In contrast to previous experiments, where elastic turbulence was investigated in channel-like geometries $[4,9,10]$, only little is known about the microscopic transport mechanisms of non-Newtonian fluids through more complex structures. This is even more surprising, since viscoelastic flow through porous media is frequently encountered in technologically relevant systems such as polymer or micellar solutions [11,12], blood [13], and colloidal suspensions [14]. It has been shown that flow of non-Newtonian liquids through porous structures can be characterized by effective macroscopic flow properties, such as an apparent viscosity, which differs significantly from the bulk properties of the fluid [15-20]. It can be expected that under conditions typically found in porous media, where the shear rate is a strongly heterogeneous quantity, elastic turbulent flow may even occur at the pore scale, thus affecting the flow resistance and the hydrodynamic dispersion, i.e. the transport of particles, molecules and immiscible fluids.

In this letter we experimentally investigate the transport properties of micron-sized tracer particles in a semi-diluted polyacrylamide (PAAm) solution flowing through a periodically patterned microarray. We demonstrate the occurrence of elastic turbulent flow for a wide range of Wi. The measured particles' transversal dispersion can be described by an effective dispersion coefficient $D_{\perp}$, which increases non-linearly with $\mathrm{Wi}$ and exceeds the corresponding value for laminar flow by several orders of 


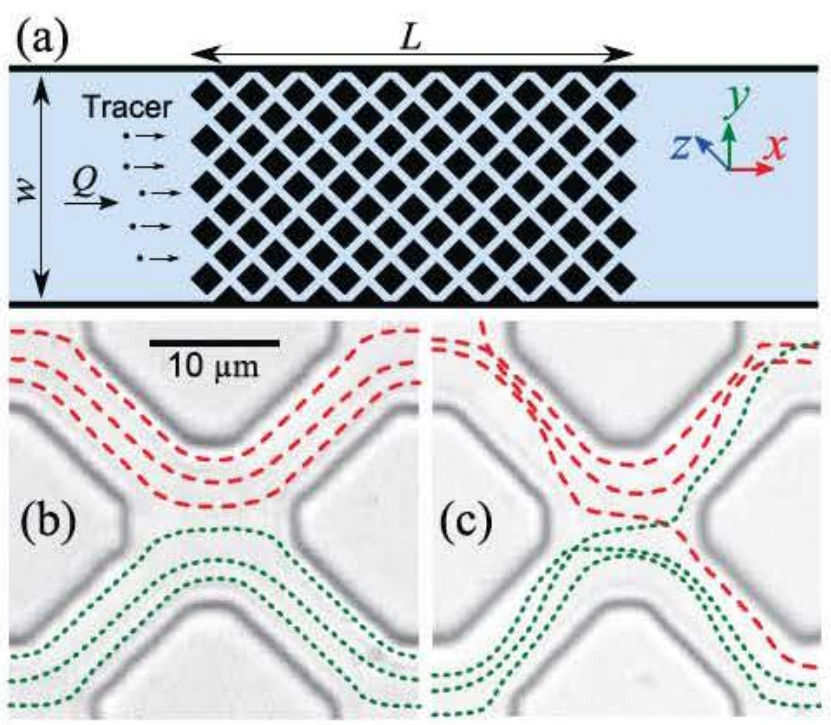

Fig. 1: (Color online) (a) Sketch of the microfluidic device used in our experiments. After injection of a diluted aqueous PAAm solution loaded with a small amount of tracer particles, a fluid flow $Q$ is imposed from left to right. (b) and (c): microscopic images of the structure with typical particle trajectories for (b) laminar flow of water at $\operatorname{Re}=6 \times 10^{-4}(\bar{u}=100 \mu \mathrm{m} / \mathrm{s})$ and (c) flow of PAAm in the regime of elastic turbulence at $\mathrm{Wi}=395, \operatorname{Re}<10^{-4}(\bar{u}=110 \mu \mathrm{m} / \mathrm{s})$ (see supplementary videos Video1.mp4 and Video2.mp4).

magnitude. In contrast to laminar flow, the flow pattern becomes asymmetric at high Wi due to the viscoelastic properties of the fluid and the shear rate depends nonlinearly on the flow rate, affecting the Wi-dependence of $D_{\perp}$.

Experiment. - Our experiments were performed in microfluidic channels of width $w=550 \mu \mathrm{m}$, length $L=$ $2.5 \mathrm{~mm}$ and height $h=7 \mu \mathrm{m}$ fabricated by soft lithography [21]. The central part of the channel was patterned with a periodic array of squares of sidelength $a=16 \mu \mathrm{m}$ and lattice constant $l=25 \mu \mathrm{m}$, which is rotated by $45^{\circ}$ relative to the flow direction (see fig. 1(a)). As a viscoleastic fluid we have used an aqueous $0.1 \%$ PAAm solution with molecular weight $M_{w}=18 \times 10^{6}$ (polyscience). The solution was deionized prior to the measurements, as rheological properties of polymer solutions are sensitive to the ion concentration [22]. To avoid aging effects [11], fresh solution was prepared $24 \mathrm{~h}$ before each measurement.

First we present a rheological characterization of the polymer solution. The typical length scale of our structure is significantly smaller than common bulk rheometer geometries. In this regime rheological differences between micro- and bulk-rheometry have been found for porous media flow, in particular a shift of the flow curve to different shear rates [20]. To account for these differences we determined the shear-dependent viscosity $\eta(\dot{\gamma})$ of the solution by active microrheology in geometries similar to our microfluidic device [23-26]. This was achieved by dragging a silica bead $(\sigma=3 \mu \mathrm{m})$ through an unpatterned

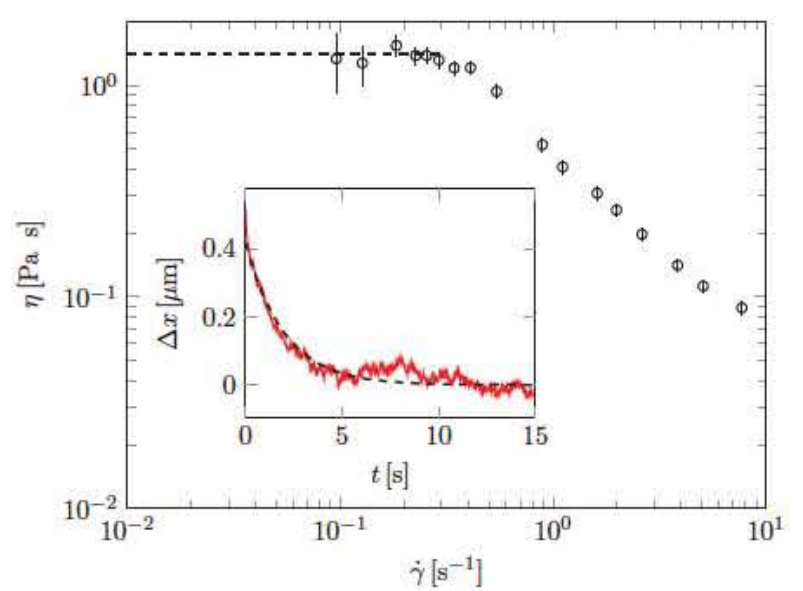

Fig. 2: (Color online) Shear-dependent viscosity of an aqueous $0.1 \%$ PAAm solution at $21^{\circ} \mathrm{C}$ determined from active microrheology. The dashed line corresponds to the zero-shear viscosity 1.4 Pas. Inset: relaxation curve of the particle position. The dashed line corresponds to the exponential fit from which the relaxation time $\lambda=2 \mathrm{~s}$ is obtained.

sample cell of $h=7 \mu \mathrm{m}$ height using a scanning optical tweezer. From the particle's response to the optical force, we obtained the shear-dependent effective viscosity (fig. 2). For small shear rates we observe a plateau which yields a zero-shear viscosity of 1.4 Pas (dashed line). As expected, for shear rates $\dot{\gamma}>3 \times 10^{-1} \mathrm{~s}^{-1}$ we observe a strong shear thinning [9]. In addition, we determined the polymer relaxation time $\lambda$. In these experiments, we first applied a constant optical dragging force to the particle $\left(\dot{\gamma} \sim 0.3 \mathrm{~s}^{-1}\right)$ and then suddenly switched the laser off. Then, the previously compressed polymers release their stress, which leads to a relaxing motion of the particle opposite to the direction of the driving force. Eventually, the particle equilibrates, leading finally to a diffusive motion (inset of fig. 2). As can be seen by the dashed line, this relaxation process can be well fitted to an exponential decay from which we determine the relaxation time to be $\lambda=2 \mathrm{~s}$.

For the particle velocimetry experiments a small amount of deionized latex tracer particles with diameter $\sigma=$ $1.3 \mu \mathrm{m}$ and $3 \mu \mathrm{m}$ was added to the solvent to visualize the flow. The microfluidic device is connected to a motorized syringe and a hydrostatic pressure reservoir to adjust the flow rate $Q$, which has been varied between 0 and $2 \mathrm{nl} / \mathrm{s}$ in our experiments. The particle motion is observed using a video microscope and a high-speed camera (Mikrotron), which allows us to record videos with frame rates up to $506 \mathrm{fps}$ at a resolution of $1280 \times 1024$ pixels covering a field of view of $350 \times 280 \mu \mathrm{m}^{2}$. This enables us to track particles with velocities up to $500 \mu \mathrm{m} / \mathrm{s}$ at a sub- $\mu \mathrm{m}$ accuracy using standard particle tracking algorithms [27-29].

Since the shear rate spatially varies in structures with non-homogeneous flows, the relevant Wi corresponds to the maximum shear rate magnitude. For our samples, Wi becomes maximal within the channel throats which have an almost quadratic cross-section $9 \times 7 \mu \mathrm{m}^{2}$. For 

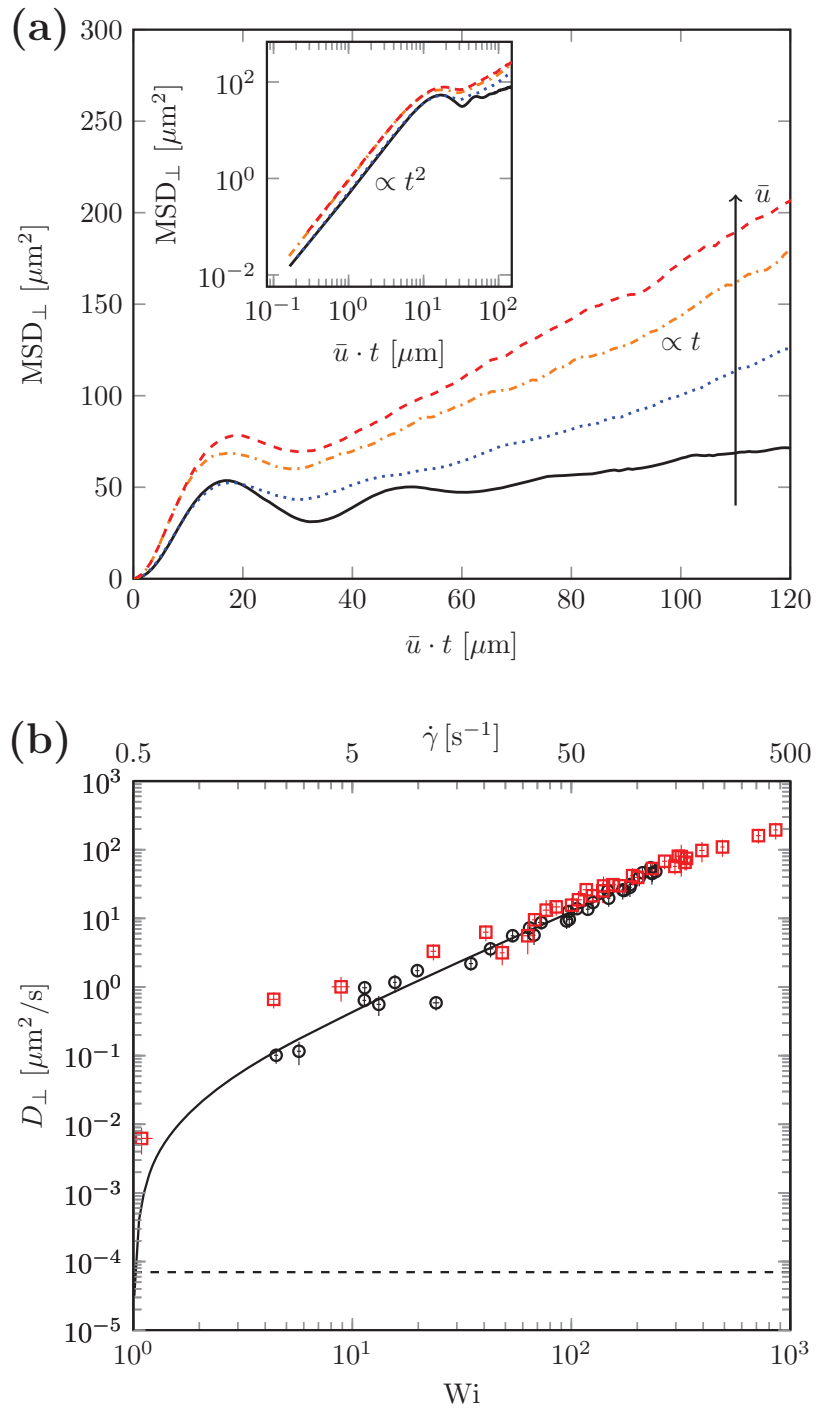

Fig. 3: (Color online) (a) Transversal mean squared displacement $\mathrm{MSD}_{\perp}$ for increasing average particle velocity $\bar{u}=$ $16,41,73,113 \mu \mathrm{m} / \mathrm{s}$ of particles with diameter $\sigma=3 \mu \mathrm{m} v s$. $\bar{u} \cdot t$. The inset shows the ballistic short-time behavior of the $\mathrm{MSD}_{\perp}$. (b) Dispersion coefficient $D_{\perp}$ vs. Wi and $\dot{\gamma}$, respectively, for $1.3 \mu \mathrm{m}(\bigcirc)$ and $3 \mu \mathrm{m}$ tracers $(\square)$. The dashed line marks the thermal diffusion coefficient at equilibrium $D_{0}=$ $(7 \pm 3) \times 10^{-5} \mu \mathrm{m}^{2} / \mathrm{s}$ (determined from passive microrheology for $\sigma=3 \mu \mathrm{m}$.). The solid curve corresponds to a power law with exponent $\alpha=1.4$ and $\mathrm{Wi}_{c}=1$.

our geometry, the maximum shear rate is given by $\dot{\gamma} \simeq 6.75 \cdot \bar{v}_{\max } / h$, where $\bar{v}_{\max }$ is the maximum velocity of the flow field $\overline{\mathbf{v}}(x, y)$ (averaged over the $z$-direction), which is obtained from the locally averaged particle velocities $\mathbf{u}(x, y)$ (see footnote ${ }^{1}$ ) [28,29]. The numerical prefactor is determined from the analytical solution for laminar flow in a rectangular pipe [30]. From these estimates we are able to obtain $\mathrm{Wi}$ as a function of the

\footnotetext{
${ }^{1}$ It has been shown in laminar flows that $\bar{u}$ is larger for smaller particles, due to (finite) size effects [29]. For laminar flow $\bar{v}=c_{d} \cdot \bar{u}$ and $c_{d}$ is a size-dependent factor in the range $1-1.5$ that can be determined from a separate calibration.
}

shear rate, i.e. the flow velocity, the latter being varied in the range $\bar{v}=0-400 \mu \mathrm{m} / \mathrm{s}$ which corresponds to shear rates $\dot{\gamma}=0-500 \mathrm{~s}^{-1}$. This yields Weissenberg numbers in the range $\mathrm{Wi}=0-900$. It should be mentioned that in the shear-thinning regime the effective relaxation time $\tau_{\text {eff }}$ can depend on $\dot{\gamma}$, which is typically taken into account in bulk rheology. However, a measurement of $\tau_{\text {eff }}$ is not feasible in our microfluidic device, where the viscosity and the relaxation time are spatially varying quantities and are therefore not identical to, e.g., bulk and channel geometries. As a consequence, we explicitly mention the corresponding shear rates to allow for a comparison with bulk measurements, where different definitions for Wi are used.

Results and discussion. - The qualitative difference between laminar flow and elastic turbulence is illustrated in figs. 1(b), (c) where we show typical particle trajectories for low Reynolds number flow of water and elastic turbulent flow at $\mathrm{Wi}=395$ (see also supplementary videos Video1.mp4 and Video2.mp4). For laminar flow the particles follow clearly separated streams in the upper and lower part of the primitive cell and fluctuations can only be caused by thermal diffusion. In contrast, for elastic turbulent flow the particle trajectories become more irregular and frequent intersections of trajectories and particle exchange between adjacent streams are observed. Quantitative information about the particle motion is obtained from the corresponding mean squared displacement. Due to the limited field of view, the longitudinal component does not approach its asymptotic behavior even for laminar flows. Therefore, we have calculated the transversal component of the mean squared displacement $\operatorname{MSD}_{\perp}(t)=\left\langle(y(t+\tau)-y(\tau))^{2}\right\rangle$ averaged over more than $10^{3}$ particles. Figure $3(\mathrm{a})$ shows the $\mathrm{MSD}_{\perp}$ for increasing average particle velocities $\bar{u}$ for $\sigma=3 \mu \mathrm{m}$ tracer particles vs. the time $t$ (rescaled by $\bar{u}$ ).

At short times, all $\mathrm{MSD}_{\perp} \mathrm{s}$ are proportional to $t^{2}$ due to the drift of the particles in the periodic flow pattern (see inset). In contrast, at long times, a linear behavior proportional to $t$ is observed. Such an effective diffusive long-time behavior has been also found in weakly turbulent flow of Newtonian liquids [31]. The intermediate oscillatory time dependence of the $\mathrm{MSD}_{\perp}$ is a result of the periodic structure. Accordingly, the first maximum and minimum at $\bar{u} \cdot t \approx 18 \mu \mathrm{m}$ and $36 \mu \mathrm{m}$, respectively correspond to the half and full period of the porous structure.

From the linear part of the $\mathrm{MSD}_{\perp}$ we obtain the transversal dispersion coefficient $D_{\perp}$ (the slope corresponds to $2 D_{\perp}$ ), which is plotted in fig. $3(\mathrm{~b})$ as a function of Wi and the corresponding $\dot{\gamma}$ for particles with $\sigma=3 \mu \mathrm{m}$ and $\sigma=1.3 \mu \mathrm{m}$. Apparently, the dispersion coefficient is rather independent of $\sigma$ and strongly increases above $\mathrm{Wi} \approx 1$. For instance, at $\mathrm{Wi}=857\left(\mathrm{Re} \approx 10^{-3}\right), D_{\perp}$ is more than 6 orders of magnitude larger than the thermal diffusion coefficient at equilibrium $D_{0}$ (dashed line). Such a notable dependence of $D_{\perp}$ on $\mathrm{Wi}$ is in good agreement with the occurrence of elastic turbulent flows in the 


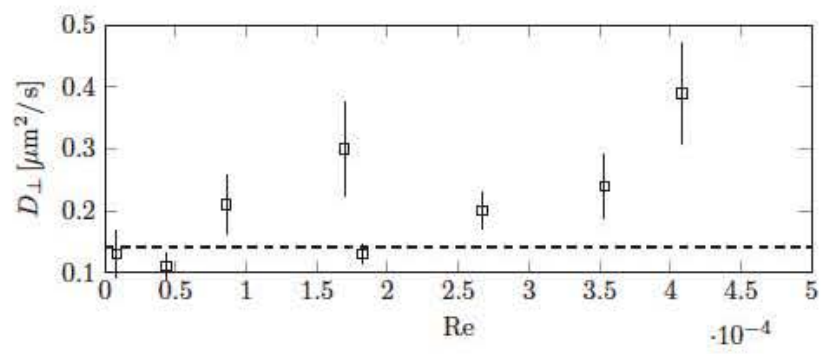

Fig. 4: Transversal dispersion coefficient $D_{\perp}$ for $\sigma=3 \mu \mathrm{m}$ latex particles in laminar flow of water. The dashed line corresponds to the diffusion coefficient at equilibrium $D_{0}\left(21^{\circ} \mathrm{C}\right)$. No significant increase of $D_{\perp}$ is observed within two orders of magnitude in $\mathrm{Re}$.
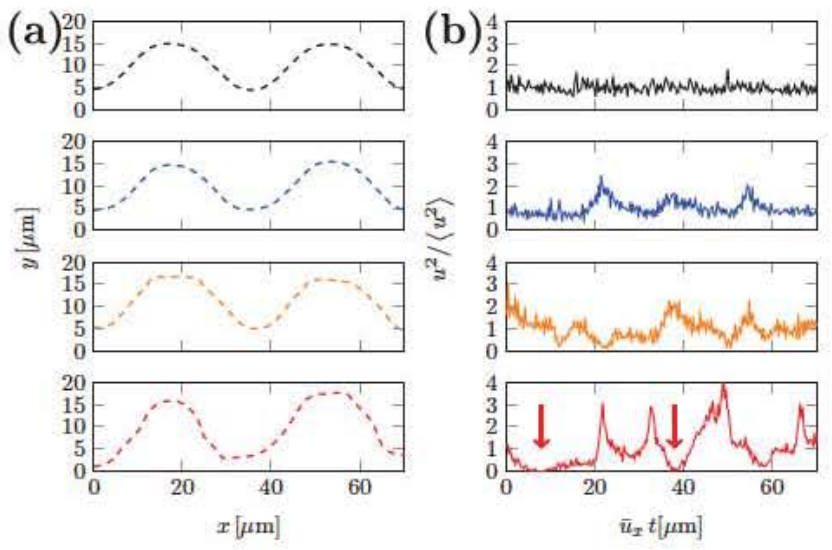

Fig. 5: (Color online) (a) Measured trajectories for Wi = $1,9,108,857$ (from top to bottom). The flow direction is from left to right. (b) Corresponding squared velocity magnitudes $u^{2} /\left\langle u^{2}\right\rangle$. The arrows point at a temporary particle halt.

viscoelastic liquid [8]. This has been confirmed by corresponding measurements in a Newtonian liquid. As shown in fig. 4 , where we plot $D_{\perp}$ of $\sigma=3 \mu \mathrm{m}$ latex particles in water, only small changes of $D_{\perp}$ compared to the microscopic diffusion coefficient $D_{0}\left(0.14 \mu \mathrm{m}^{2} / \mathrm{s}\right.$, dashed line $)$ are observed for increasing Re. This effect can attributed to mechanical dispersion which should indeed slightly contribute to the dispersion in porous media [32,33].

To motivate a heuristic description for the dependence of $D_{\perp}$ on $\mathrm{Wi}$, we make use of the findings that i) dispersion coefficients in laminar flow often show a power-law dependence on the Péclet and Reynolds number [34] and ii) that in macroscopic experiments with viscoelastic liquids a critical Weissenberg number $\mathrm{Wi}_{c} \simeq 1$ is observed (corresponding to a critical $\dot{\gamma}_{c}=0.5 \mathrm{~s}^{-1}$ ), which marks the onset of elastic turbulence $[1,4,7,8]$. Accordingly, we fitted the data in fig. $3(\mathrm{~b})$ to $D_{\perp}=D_{0}+c \cdot\left(\mathrm{Wi}-\mathrm{Wi}_{c}\right)^{\alpha}$ and find good agreement with the experimental data over two decades with $c=0.02 \mu \mathrm{m}^{2} / \mathrm{s}, \alpha=1.4$ (solid line). Considering our definition of Wi, this scaling behavior is essentially a function of $\dot{\gamma}$, as $\tau$ is constant for all data points.

The asymptotic diffusive behavior of the particles should also be reflected by increased particle velocity
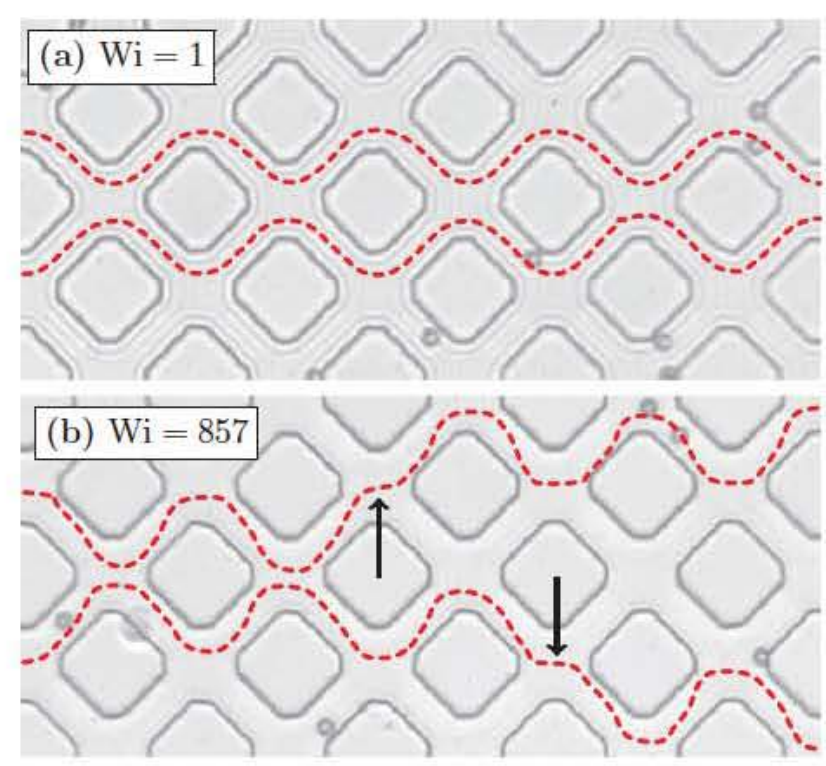

Fig. 6: (Color online) (a) Two exemplary trajectories that illustrate the particle motion close to $\mathrm{Wi}_{c}$. The flow direction is from left to right. (b) Two exemplary trajectories that show transversal hopping between streamlines far above $\mathrm{Wi}_{c}$. The arrows point at the pores where particles cross to an adjacent stream.

fluctuations for $\mathrm{Wi}>\mathrm{Wi}_{c}$. This can be demonstrated by comparing typical trajectories at $\mathrm{Wi}=1,9,108,857$, which are shown in fig. 5(a) (from top to bottom) for particles with $\sigma=3 \mu \mathrm{m}$. Although all trajectories closely follow the periodic flow field, the velocity fluctuations significantly increase with Wi. This is shown in fig. 5(b), where we plot the corresponding normalized squared particle velocity $u^{2}$. With increasing Wi we observe stronger accelerations, which can even result in temporary particle halts, as indicated by the arrow in fig. 5(b).

Particle velocity fluctuations are also responsible for streamline crossing, which eventually leads to enhanced dispersion along the $y$-direction. In case of laminar flow, the transversal displacement while a particle travels a given distance in $x$-direction, decreases with increasing flow rate. In contrast, here we observe a strong increase of lateral particle displacements for increasing Wi. This is further illustrated in figs. 6(a), (b) where we plotted characteristic trajectories of particles with $\sigma=3 \mu \mathrm{m}$ for $\mathrm{Wi}=1$ and 857 . In contrast to $\mathrm{Wi}=1$ where most particle trajectories remain within a single stream (leading to a rather periodic motion), for $\mathrm{Wi}=857$ frequent particle crossing between adjacent streams is observed (arrows in fig. 6(b)). For $\mathrm{Wi}=857$, the probability for such particle crossings within a single primitive cell is about $8 \%$ while for $\mathrm{Wi}=1$ the corresponding value is close to 0 .

Our experiments show that elastic turbulence affects the particle motion in non-Newtonian liquids similar to thermal fluctuations. However, while in laminar flow the average velocity field is deterministic and proportional to $Q$, viscoelastic fluids exhibit non-linear velocity patterns $[2,4,35]$. In the presence of porous media, this 

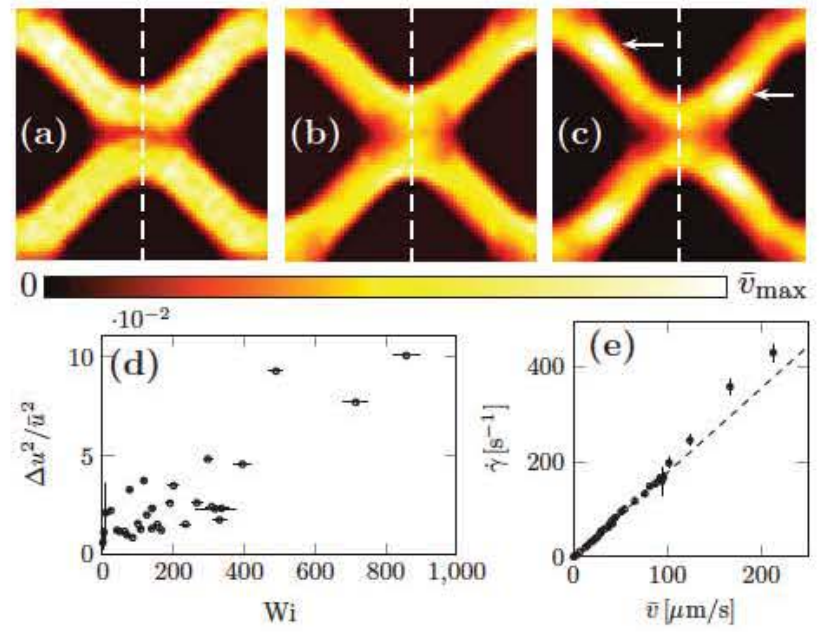

Fig. 7: (Color online) Averaged velocity field $\overline{\mathbf{v}}(x, y)$ (velocity magnitude given by grayscale) for (a) $\mathrm{Wi}=1$, (b) $\mathrm{Wi}=108$ and (c) $\mathrm{Wi}=857$. The flow direction is from left to right. (d) Mean squared difference of a flow field and its mirror image as a function of Wi. (e) Shear rate $\dot{\gamma}$ determined from the velocity fields as a function of $\bar{v}$.

leads to time- and space-dependent shear rates and a nonlinear dependence on the average velocity, both affecting the particle dispersion in such systems. To illustrate how the non-linearities of the flow field affect the maximum shear rate in our system, we show the average velocity field determined from a temporal average over the local particle velocities mapped to the primitive cell of the structure (figs. 7(a)-(c)). Close to $\mathrm{Wi}_{c}$, the streams in the lower and upper half of the primitive cell are symmetric and separated by a region of vanishing velocity in the center (fig. 7(a)). These streams become broadened with growing Wi and eventually overlap in the center of the primitive cell as illustrated for $\mathrm{Wi}=108$ in fig. 7(b). A closer analysis reveals that the flow field becomes asymmetric relative to the vertical symmetry axis (dashed vertical lines) of the primitive cell, at high Wi. This asymmetry can be quantified by the mean squared difference between the velocity field and its mirror image relative to the vertical symmetry axis $\Delta u^{2}$. As illustrated in fig. 7 (d) the asymmetry is almost zero up to $\mathrm{Wi} \approx 200$ and increases only slowly at intermediate Wi. For Wi $\gtrsim 400, \Delta u^{2}$ rapidly increases due to the formation of high velocity regions (see arrows in fig. 7(c)). This non-linear instability is attributed to the elastic forces in the system [35] and becomes relevant at high Wi when the whole system is strongly sheared. Consequently, the determined shear rate at high Wi is no longer proportional to the average flow velocity $\bar{v}$ but grows stronger than linearly (see fig. 7(e)).

Apart from this asymmetry, no large-scale instabilities have been found in the flow. This can be radically different in random porous geometries. Although we have also observed elastic turbulence in random porous structures (data not shown) made of randomly overlapping monodisperse circles (see [36] for details), the rapidly diverging correlation length makes it difficult to obtain dispersion coefficients from particle trajectories. In contrast to periodic structures, random porous media typically also possess stagnant parts where the flow almost vanishes. Such stagnant parts are known to strongly influence the particle transport and dispersion [37-39]. Moreover, it has been observed that stagnant parts lead to the formation of vortices in viscoelastic fluids [40]. Similar to inertial turbulent flow, where trapping of particles in vortices leads to superdiffusive dispersion due to Lévy flights [31], we expect that such effects may also arise for elastic turbulence.

To further investigate the scaling of $D_{\perp}$ in the shearthinning regime a shear-dependent Weissenberg number might be considered which could result in a different Wi dependence for large shear rates compared to our definition of Wi. Ideally this requires a shear-dependent measurement of the relaxation time within the microfluidic geometry. Another open question is the influence of the particle size on the dispersion coefficient. While we find no significant contribution of the particle size to the dispersion, in the limits $\sigma \rightarrow 0$ and $\sigma \rightarrow h$, the particle size must be considered. This question is closely related to the microscopic structure of the entangled polymers in the flow. While this structure cannot be obtained by conventional microscopy, techniques such as cryogenic transmission electron microscopy could be used to obtain this information $[41,42]$.

Summary. - In summary, we demonstrated elastic turbulent dispersion of finite-sized tracer particles in microfluidic porous media. We conclude from the asymptotic behavior of the $\mathrm{MSD}_{\perp}$ that dispersion in elastic turbulent flows can be characterized in terms of an effective dispersion coefficient. Due to strongly enhanced velocity fluctuations $D_{\perp}$ increases significantly with Wi. We find a non-linear dependence of $D_{\perp}$ on Wi and observe a data collapse for different-sized particles, indicating that $D_{\perp}$ is mainly determined by $\mathrm{Wi}$ and only weakly dependent on the thermal diffusion. In addition to the turbulent velocity fluctuations, we observe a non-linear asymmetry in the averaged flow field that occurs for high Wi and leads to a non-linear dependence of the shear rate on the flow.

We would like to thank YUJIE LI, HANS-JÜRGEN KÜMMERER and GERD E. SCHRÖDER-TURK for helpful discussions.

\section{REFERENCES}

[1] Groisman A. and Steingerg V., Nature, 405 (2000) 53.

[2] Bird R. B., Armstrong R. C. and Hassager O., Dynamics of Polymeric Liquids, Vol. 1, Fluid Mechanics (John Wiley \& Sons, Inc.) 1987.

[3] Dealy J., Rheol. Bull., 79 (2010) 14.

[4] ZILZ J. et al., J. Fluid Mech., 712 (2012) 203. 
[5] Groisman A. and Steinberg V., Phys. Rev. Lett., 86 (2001) 934.

[6] Beaumont J. et al., Soft Matter, 9 (2013) 735.

[7] Groisman A. and Steinberg V., Nature, 410 (2001) 905.

[8] Burghelea T., Segre E., Bar-Joseph I., Groisman A. and Steinberg V., Phys. Rev. E, 69 (2004) 066305.

[9] Bonn D., Ingremeau F., Amarouchene Y. and KelLAY H., Phy. Rev. E, 84 (2011) 045301.

[10] Pan L., Morozov A., Wagner C. and Arratia P., Phys. Rev. Lett., 110 (2013) 174502.

[11] Kulicke W.-M., Kötter M. and Gräger H., Drag reduction phenomenon with special emphasis on homogeneous polymer solutions, in Polymer Characterization/Polymer Solutions (Springer) 1989, pp. 1-68.

[12] Rojas M. R., Müller A. J. and Sáez A. E., J. Colloid Interface Sci., 326 (2008) 221.

[13] Merrill E. W., Physiol. Rev., 49 (1969) 863.

[14] Russel W. B., Saville D. A. and Schowalter W. R., Colloidal Dispersions (Cambridge University Press) 1992.

[15] Bird R. B., Stewart W. E. and Lightfoot E. N., Transport Phenomena (Wiley, New York) 1960.

[16] Chauveteau G., J. Rheol., 26 (1982) 111.

[17] Lake L. W., Enhanced Oil Recovery (Prentice Hall Inc., Old Tappan, NJ) 1989.

[18] Sorbie K., Clifford P. and Jones E., J. Colloid Interface Sci., 130 (1989) 508.

[19] Lopez X., Valvatne P. H. and Blunt M. J., J. Colloid Interface Sci., 264 (2003) 256.

[20] Perrin C. L., Tardy P. M., Sorbie K. S. and CrawShaw J. C., J. Colloid Interface Sci., 295 (2005) 542.

[21] Whitesides G. and Stroock A., Phys. Today, 54, issue No. 6 (2001) 42.

[22] Sun Y., Saleh L. and BAi B., Measurement and impact factors of polymer rheology in porous media, in Rheology, edited by ViCEnTE D. J. D. (Intech) 2012, pp. 187-202.

[23] DePuit R. J., Khair A. S. and Squires T. M., Phys. Fluids, 23 (2011) 063102.
[24] Sriram I., Meyer A. and Furst E. M., Phys. Fluids, 22 (2010).

[25] Wilson L. G. and Poon W. C., Phys. Chem. Chem. Phys., 13 (2011) 10617.

[26] Gomez-Solano J. R. and Bechinger C., submitted to EPL (2014).

[27] Crocker J. C. and Grier D. G., J. Colloid Interface Sci., 179 (1996) 298.

[28] Santiago J. G., Wereley S. T., Meinhart C. D., Beebe D. J. and Adrian R. J., Exp. Fluids, 25 (1998) 316.

[29] Scholz C., Wirner F., Li Y. and Bechinger C., Exp. Fluids, 53 (2012) 1327.

[30] Bruus H., Theoretical Microfluidics (Oxford University Press) 2007.

[31] Weeks E. R. and Swinney H. L., http://www.physics. emory.edu/faculty/weeks/abs/nst98.html (1998).

[32] Fried J. and Combarnous M., Adv. Hydrosci., 7 (1971) 169.

[33] Han N.-W., Bhakta J. and Carbonell R., AiChE J., 31 (1985) 277.

[34] Bear J., Dynamics of Fluids in Porous Media (Dover Publications) 2013.

[35] Arratia P. E., Thomas C. C., Diorio J. and Gollub J. P., Phys. Rev. Lett., 96 (2006) 144502.

[36] Scholz C. et al., Phys. Rev. Lett., 109 (2012) 264504.

[37] Gist G., Thompson A., Katz A. and Higgins R., Phys. Fluids, 2 (1990) 1533.

[38] Bijeljic B., Mostaghimi P. and Blunt M. J., Phys. Rev. Lett., 107 (2011) 204502.

[39] Wirner F., Scholz C. and Bechinger C., Phys. Rev. E, 90 (2014) 013025.

[40] Кномамi B. and Moreno L. D., Rheol. Acta, 36 (1997) 367.

[41] Vasudevan M. et al., Nat. Mater., 9 (2010) 436.

[42] Cardiel J. J. et al., Proc. Natl. Acad. Sci. U.S.A., 110 (2013) E1653. 